PROCEEDINGS OF THE

AMERICAN MATHEMATICAL SOCIETY

Volume 136, Number 8, August 2008, Pages 2711-2717

S 0002-9939(08)09281-2

Article electronically published on April 3, 2008

\title{
$A$-IDENTITIES FOR THE GRASSMANN ALGEBRA: THE CONJECTURE OF HENKE AND REGEV
}

\author{
DIMAS JOSÉ GONÇALVES AND PLAMEN KOSHLUKOV
}

(Communicated by Birge Huisgen-Zimmermann)

\begin{abstract}
Let $K$ be an algebraically closed field of characteristic 0 , and let $E$ be the infinite dimensional Grassmann (or exterior) algebra over $K$. Denote by $P_{n}$ the vector space of the multilinear polynomials of degree $n$ in $x_{1}, \ldots$, $x_{n}$ in the free associative algebra $K(X)$. The symmetric group $S_{n}$ acts on the left-hand side on $P_{n}$, thus turning it into an $S_{n}$-module. This fact, although simple, plays an important role in the theory of PI algebras since one may study the identities satisfied by a given algebra by applying methods from the representation theory of the symmetric group. The $S_{n}$-modules $P_{n}$ and $K S_{n}$ are canonically isomorphic. Letting $A_{n}$ be the alternating group in $S_{n}$, one may study $K A_{n}$ and its isomorphic copy in $P_{n}$ with the corresponding action of $A_{n}$. Henke and Regev described the $A_{n}$-codimensions of the Grassmann algebra $E$, and conjectured a finite generating set of the $A_{n}$-identities for $E$. Here we answer their conjecture in the affirmative.
\end{abstract}

\section{INTRODUCTION}

Let $K$ be a fixed algebraically closed field of characteristic 0 ; all algebras, vector spaces and modules we consider will be over this field. Denote by $E$ the Grassmann algebra of the infinite dimensional vector space $V$ over $K$. If $V$ has a basis $e_{1}$, $e_{2}, \ldots$, then $E$ has a basis consisting of 1 and the elements $e_{i_{1}} e_{i_{2}} \ldots e_{i_{k}}$ where $i_{1}<i_{2}<\cdots<i_{k}, k \geq 1$. The multiplication in $E$ is induced by $e_{i} e_{j}=-e_{j} e_{i}$ for all $i$ and $j$. Thus the centre $E_{0}$ of $E$ is the span of 1 and all elements $e_{i_{1}} e_{i_{2}} \ldots e_{i_{k}}$ with even $k$. Let $E_{1}$ be the span of all such elements with odd $k$. Then the elements of $E_{1}$ anticommute. It is an immediate observation that for every $a, b, c \in E$ one has $[a, b] \in E_{0}$ and therefore $[a, b, c]=0$. We suppose all commutators to be left normed; that is, $[a, b, c]$ stands for $[[a, b], c]$ and so on.

Let $K(X)$ be the free associative algebra with 1 , freely generated over $K$ by the set of variables $X=\left\{x_{1}, x_{2}, \ldots\right\}$. A polynomial $f \in K(X)$ is a polynomial identity, or simply an identity for the algebra $R$ if $f\left(r_{1}, \ldots, r_{m}\right)=0$ whenever $r_{i} \in R$. The set of all identities for $R$ is an ideal $T(R)$; it is the $T$-ideal of $R$. Since char $K=0$ one has that $T(R)$ is generated as a $T$-ideal by its multilinear polynomials. Denote by $P_{n}$ the set of all multilinear polynomials of degree $n$ in $x_{1}, \ldots, x_{n}$. Then $T(R)$ is generated by all $T(R) \cap P_{n}, n \geq 1$. The vector space $P_{n}$ has a basis consisting

Received by the editors April 23, 2007, and, in revised form, June 19, 2007.

2000 Mathematics Subject Classification. Primary 16R10; Secondary 16R40, 16R50.

The first author was supported by a Ph.D. grant from PRPG, UNICAMP.

The second author was partially supported by grants from CNPq (No. 302655/2005-0), and from FAPESP (No. 2004/13766-2 and 2005/60337-2).

(C)2008 American Mathematical Society 2711

Reverts to public domain 28 years from publication 
of $\left\{x_{\sigma(1)} \ldots x_{\sigma(n)} \mid \sigma \in S_{n}\right\}$. Here and in what follows $S_{n}$ is the symmetric group acting on $1,2, \ldots, n$, and $A_{n}$ is the alternating subgroup of $S_{n}$.

The (left) regular $S_{n}$-module $K S_{n}$ is isomorphic to $P_{n}$ as follows: if $\sigma \in S_{n}$, then $\sigma \mapsto x_{\sigma(1)} \ldots x_{\sigma(n)}$. Thus one may identify $K S_{n}$ with $P_{n}$ as $S_{n}$-modules. Since $K A_{n} \subseteq K S_{n}$, one considers $P_{n}^{A}$, the image of $K A_{n}$ in $P_{n}$. The elements of $P_{n}^{A}$ are called $A$-polynomials. If $R$ is any PI algebra with $T$-ideal $T=T(R)$, then the $A$-identities of $R$ are the polynomials in $T \cap P_{n}^{A}, n \geq 1$. It was pointed out in 2] that every PI algebra $R$ satisfies some $A$-identity: if $f\left(x_{1}, \ldots, x_{n}\right)$ is a multilinear identity for $R$, then $f\left(x_{1} x_{2}, \ldots, x_{2 n-3} x_{2 n-2}, x_{2 n-1}\right)$ will be an $A$-identity for $R$. We recall that in 1 the authors described the module structure of the group algebra $K A_{n}$ in terms of Young tableaux and related it to the one of $K S_{n}$; see [1 and its references for further information concerning the relation between $A_{n}$ and PI algebras.

Letting $R$ be as above, one defines the $n$-th codimension $c_{n}(R)$ of $R$ as follows: $c_{n}(R)=\operatorname{dim} P_{n} /\left(T \cap P_{n}\right)$. The $n$-th $A$-codimension is $c_{n}^{A}(R)=\operatorname{dim} P_{n}^{A} /\left(T \cap P_{n}^{A}\right)$. It was proved in [3] that for the Grassmann algebra one has $c_{n}(E)=2^{n-1}$. It was also proved in [3] that $T(E)$ is generated as a $T$-ideal by the single polynomial $\left[x_{1}, x_{2}, x_{3}\right]$, and a detailed description of the module structure of the $S_{n}$-module $P_{n}(E)=P_{n} /\left(T(E) \cap P_{n}\right)$ was obtained as well. Furthermore one of the main results in 2] states that $c_{n}^{A}(E)=2^{n-1}-1$; see [2, Theorem 2.2].

We recall that since $E$ satisfies the identity $\left[x_{1}, x_{2}, x_{3}\right]$, then it also satisfies the $A$-identity of degree five $\left[x_{1} x_{2}, x_{3} x_{4}, x_{5}\right]$. But in [2, Example 1.1] it was shown that $E$ satisfies the $A$-identity

$$
p=p\left(x_{1}, x_{2}, x_{3}, x_{4}\right)=\left[x_{1}, x_{2} x_{3}\right] x_{4}-x_{4}\left[x_{1}, x_{3} x_{2}\right]
$$

and it is of minimal degree. Furthermore in [2, Conjecture 1.2] the authors conjectured that the above polynomial generates all $A$-identities of $E$ in the following strong sense.

Conjecture 1. Let $\sigma \in A_{n}$ and $0 \leq r \leq n-4$; put $p_{r, \sigma}=x_{\sigma(1)} \ldots x_{\sigma(r)}$ and $q_{r, \sigma}=x_{\sigma(r+5)} \ldots x_{\sigma(n)}$. Define $f_{r, \sigma}$ as the polynomial

$$
p_{r, \sigma}\left(\left[x_{\sigma(r+1)}, x_{\sigma(r+2)} x_{\sigma(r+3)}\right] x_{\sigma(r+4)}-x_{\sigma(r+4)}\left[x_{\sigma(r+1)}, x_{\sigma(r+3)} x_{\sigma(r+2)}\right]\right) q_{r, \sigma} .
$$

Then the polynomials $f_{r, \sigma}$ span all A-identities for $E$. (Observe that all $f_{r, \sigma}$ are A-identities.)

In this paper we give an affirmative answer to the above conjecture.

Let $V(n)$ be the span of all polynomials $f_{r, \sigma}, 0 \leq r \leq n-4$, and $\sigma \in A_{n}$. Our main result is the following theorem.

Theorem 2. If $n \geq 4$, then $\operatorname{dim} P_{n}^{A} / V(n)=2^{n-1}-1$.

We observe that Theorem 2 implies immediately the affirmative answer of Conjecture 1 Indeed $V(n) \subseteq P_{n}^{A} \cap T(E)$, and if for some $n$ the inclusion were proper, then one would have $\operatorname{dim} P_{n}^{A} / V(n)>\operatorname{dim} P_{n}^{A} /\left(P_{n}^{A} \cap T(E)\right)=c_{n}^{A}(E)$. But the latter $A$-codimension equals $2^{n-1}-1$ according to [2, Theorem 2.2].

\section{Proof of Theorem 2}

We prove the theorem by induction on $n$. First we establish some properties of $V(n)$. We consider the quotient module $P_{n}^{A} / V(n)$. We shall use the letters $y$ for 
the images of $x$ in the quotient. That is,

$$
y_{\sigma(1)} y_{\sigma(2)} \ldots y_{\sigma(n)}=x_{\sigma(1)} x_{\sigma(2)} \ldots x_{\sigma(n)}+V(n) .
$$

When dealing with the monomial $m=y_{\sigma(1)} y_{\sigma(2)} \ldots y_{\sigma(n)}$, if we are interested only in some submonomial $m^{\prime}$, say $m=m_{1} m^{\prime} m_{2}$, then we shall substitute $m_{1}$ and $m_{2}$ by $*$, that is $m=* m^{\prime} *$. Observe that $*$ may stand for the empty monomial as well. Thus from the basic identity (1) we have:

Lemma 3. The equality

$$
* y_{a} y_{b} y_{c} y_{d} *-* y_{d} y_{a} y_{c} y_{b} *-* y_{b} y_{c} y_{a} y_{d} *+* y_{d} y_{c} y_{b} y_{a} *=0
$$

holds in $P_{n}^{A} / V(n)$ for every $a, b, c, d$.

We note that the above equality reads

$$
m_{1} y_{a} y_{b} y_{c} y_{d} m_{2}-m_{1} y_{d} y_{a} y_{c} y_{b} m_{2}-m_{1} y_{b} y_{c} y_{a} y_{d} m_{2}+m_{1} y_{d} y_{c} y_{b} y_{a} m_{2}=0
$$

for some monomials $m_{1}$ and $m_{2}$. In what follows, in an equality all left-hand side asterisks stand for the same monomial and similarly for the right-hand side asterisks.

Lemma 4. The vector space $P_{n}^{A} / V(n)$ is spanned by monomials of the type $y_{\sigma(1)} y_{\sigma(2)} \ldots y_{\sigma(n)}$ where $y_{n}$ is at one of the last three positions. In other words either $\sigma(n-2)=n$, or $\sigma(n-1)=n$, or else $\sigma(n)=n$.

Proof. In (2), the variable $y_{a}$ appears at the first position only in the first summand. Therefore applying (2) several times to a given monomial, we represent it as a linear combination of monomials where $y_{n}$ is in one of the last three positions.

Denote by $H(i, j)$ the span of all $y_{\sigma(1)} y_{\sigma(2)} \ldots y_{\sigma(n)}$ such that $\sigma(i)=n$ and $\sigma(j)=n-2$. That is, $H(i, j)$ is spanned by the monomials where $y_{n}$ is at position $i$ and $y_{n-2}$ is at position $j$. Here we require $1 \leq i, j \leq n$ and $i \neq j$.

Lemma 5. The vector space $P_{n}^{A} / V(n)$ is the sum of its subspaces $H(i, j)$ such that $i \geq n-3$ and $j \geq n-3$.

Proof. As in the proof of the previous lemma, observe the behaviour of the variables $y_{a}$ and $y_{c}$ in (2).

Let $H=\sum_{j=n-3}^{n-1} H(n, j)+\sum_{i=n-3}^{n-1} H(i, n)$. This means that $H$ is the span of the monomials whose last position is occupied either by $y_{n}$ or by $y_{n-2}$.

Proposition 6. One has $P_{n}^{A} / V(n)=H+H(n-1, n-2)+H(n-2, n-1)$.

Proof. We shall use the equality (2) several times:

$$
* y_{a} y_{b} y_{c} y_{d} *-* y_{d} y_{a} y_{c} y_{b} *-* y_{b} y_{c} y_{a} y_{d} *+* y_{d} y_{c} y_{b} y_{a} *=0
$$

in order to eliminate $H(n-2, n-3), H(n-1, n-3), H(n-3, n-2)$ and $H(n-3, n-1)$.

Case 1. Let $m=y_{\sigma(1)} \ldots y_{\sigma(n-4)} y_{n-2} y_{n} y_{\sigma(n-1)} y_{\sigma(n)} \in H(n-2, n-3)$. We rewrite (2) as

$$
* y_{d} y_{a} y_{c} y_{b} *=* y_{a} y_{b} y_{c} y_{d} *-* y_{b} y_{c} y_{a} y_{d} *+* y_{d} y_{c} y_{b} y_{a} *
$$

and set $d=n-2, a=n, c=\sigma(n-1), b=\sigma(n)$ in it. Therefore we obtain

$$
\begin{aligned}
* y_{n-2} y_{n} y_{\sigma(n-1)} y_{\sigma(n)}=* y_{n} y_{\sigma(n)} y_{\sigma(n-1)} y_{n-2} & -* y_{\sigma(n)} y_{\sigma(n-1)} y_{n} y_{n-2} \\
& +* y_{n-2} y_{\sigma(n-1)} y_{\sigma(n)} y_{n} .
\end{aligned}
$$

All monomials from the right-hand side of the above equality lie in $H$; hence $m \in H$. 
Case 2. Let $m=y_{\sigma(1)} \ldots y_{\sigma(n-4)} y_{n} y_{n-2} y_{\sigma(n-1)} y_{\sigma(n)} \in H(n-3, n-2)$. We proceed as in case 1 , with the only difference that we exchange $n$ and $n-2$; that is, we set $d=n, a=n-2$, and repeat the argument above.

Case 3 . Let $m=y_{\sigma(1)} \ldots y_{\sigma(n-4)} y_{n-2} y_{\sigma(n-2)} y_{n} y_{\sigma(n)} \in H(n-1, n-3)$. Here we rewrite (2) as

$$
* y_{d} y_{c} y_{b} y_{a} *=-* y_{a} y_{b} y_{c} y_{d} *+* y_{d} y_{a} y_{c} y_{b} *+* y_{b} y_{c} y_{a} y_{d} *
$$

and set in it $d=n-2, c=\sigma(n-2), b=n, a=\sigma(n)$. Thus we obtain

$$
\begin{aligned}
* y_{n-2} y_{\sigma(n-2)} y_{n} y_{\sigma(n)}=-* y_{\sigma(n)} y_{n} y_{\sigma(n-2)} y_{n-2} & +* y_{n-2} y_{\sigma(n)} y_{\sigma(n-2)} y_{n} \\
& +* y_{n} y_{\sigma(n-2)} y_{\sigma(n)} y_{n-2} .
\end{aligned}
$$

As in case 1, all monomials from the right-hand side are in $H$; thus $m \in H$.

Case 4 . Now let $m=y_{\sigma(1)} \ldots y_{\sigma(n-4)} y_{n} y_{\sigma(n-2)} y_{n-2} y_{\sigma(n)} \in H(n-3, n-1)$. As was done before (see Case 2), it is sufficient to exchange $d$ and $b$ in Case 3 , that is, to set $d=n, b=n-2$.

Hence all possibilities have been considered and the proof of the proposition is complete.

Corollary 7. $P_{n}^{A} / V(n)=H+H(n-2, n-1)$.

Proof. Let $m=y_{\sigma(1)} \ldots y_{\sigma(n-4)} y_{\sigma(n-3)} y_{n-2} y_{n} y_{\sigma(n)} \in H(n-1, n-2)$. Write (2) as

$$
* y_{a} y_{b} y_{c} y_{d} *=* y_{d} y_{a} y_{c} y_{b} *+* y_{b} y_{c} y_{a} y_{d} *-* y_{d} y_{c} y_{b} y_{a} *,
$$

and set in it $a=\sigma(n-3), b=n-2, c=n, d=\sigma(n)$. Thus

$$
\begin{aligned}
* y_{\sigma(n-3)} y_{n-2} y_{n} y_{\sigma(n)}=* y_{\sigma(n)} y_{\sigma(n-3)} y_{n} y_{n-2} & +* y_{n-2} y_{n} y_{\sigma(n-3)} y_{\sigma(n)} \\
- & * y_{\sigma(n)} y_{n} y_{n-2} y_{\sigma(n-3)} .
\end{aligned}
$$

Then $m \in H+H(n-2, n-3)+H(n-2, n-1)$. But $H(n-2, n-3) \subseteq H$ as was shown in Case 1 of the proof of the last proposition. Therefore the corollary is proved.

Lemma 8. The equalities $\left[y_{1} y_{2}, y_{3}, y_{4} y_{5}\right]=0$ and $\left[y_{1} y_{2}, y_{3} y_{4}, y_{5}\right]=0$ are verified in $P_{5}^{A} / V(5)$.

Proof. The proof is a straightforward computation:

$$
\left[y_{1} y_{2}, y_{3}, y_{4} y_{5}\right]=y_{1} y_{2} y_{3} y_{4} y_{5}-y_{3} y_{1} y_{2} y_{4} y_{5}-y_{4} y_{5} y_{1} y_{2} y_{3}+y_{4} y_{5} y_{3} y_{1} y_{2} \text {. }
$$

Now we transform the second summand above using (2):

$$
\begin{aligned}
-y_{3} y_{1} y_{2} y_{4} y_{5}= & -\left(y_{4} y_{3} y_{2} y_{1}+y_{1} y_{2} y_{3} y_{4}-y_{4} y_{2} y_{1} y_{3}\right) y_{5} \\
= & -y_{4}\left(y_{5} y_{3} y_{1} y_{2}+y_{2} y_{1} y_{3} y_{5}-y_{5} y_{1} y_{2} y_{3}\right) \\
& -y_{1} y_{2} y_{3} y_{4} y_{5}+y_{4} y_{2} y_{1} y_{3} y_{5} \\
= & -y_{4} y_{5} y_{3} y_{1} y_{2}+y_{4} y_{5} y_{1} y_{2} y_{3}-y_{1} y_{2} y_{3} y_{4} y_{5}
\end{aligned}
$$

Thus $\left[y_{1} y_{2}, y_{3}, y_{4} y_{5}\right]=0$ in $P_{5}^{A} / V(5)$. The second equality follows from the first by the Jacobi identity $\left[y_{1} y_{2}, y_{3} y_{4}, y_{5}\right]=\left[y_{1} y_{2}, y_{5}, y_{3} y_{4}\right]-\left[y_{3} y_{4}, y_{5}, y_{1} y_{2}\right]$ since both right-hand side summands vanish on $P_{5}^{A} / V(5)$.

Corollary 9. If $n \geq 3$, then

$$
*\left[y_{1} y_{2}, y_{3} y_{4}, \ldots, y_{2 n-1} y_{2 n}\right] *=0 .
$$


Proof. The proof follows immediately from Lemma 8 ,

Proposition 10. Let $n \geq 4$ and let $m=y_{\sigma(1)} y_{\sigma(2)} \ldots y_{\sigma(n)}$.

a) Let $n$ be even and let for some $k, 1 \leq k \leq n / 2, \sigma(2 k-1)=n$ and $\sigma(2 k)=$ $n-2$. Then $m \in H$.

b) If $n$ is odd and if for some $k, 1 \leq k \leq(n-1) / 2, \sigma(2 k)=n$ and $\sigma(2 k+1)=$ $n-2$, then $m \in H$.

Proof. By Corollary 9 we reduce $m$ to a linear combination of monomials of the form $* y_{n} y_{n-2} y_{a} y_{b}$ and monomials in $H$. But $* y_{n} y_{n-2} y_{a} y_{b} \in H(n-3, n-2) \subseteq H$, where the last inclusion was proved in Proposition 6, Case 2.

Here we give an example. Let $m=y_{6} y_{4} y_{1} y_{2} y_{3} y_{5}$; thus in the notation of the last proposition, $n=6, k=1$. According to Corollary 9 we have $\left[y_{6} y_{4}, y_{1} y_{2}, y_{3} y_{5}\right]=0$; therefore

$y_{6} y_{4} \cdot y_{1} y_{2} \cdot y_{3} y_{5}=y_{1} y_{2} \cdot y_{6} y_{4} \cdot y_{3} y_{5}+y_{3} y_{5} \cdot y_{6} y_{4} \cdot y_{1} y_{2}-y_{3} y_{5} \cdot y_{1} y_{2} \cdot y_{6} y_{4} \cdot$

The first two summands on the right-hand side lie in $H(n-3, n-2) \subseteq H$ (recall that $n=6$ here), and the last one belongs to $H$ by definition.

Lemma 11. Let $m=* y_{a} y_{b} y_{n} y_{n-2} y_{c} * \in\left(P_{n}^{A} / V(n)\right) \backslash H$ be a monomial. Then there exist $h_{1}$ and $h_{2} \in H$ such that:

$$
m=* y_{n} y_{n-2} y_{a} y_{b} y_{c} *+h_{1} ; \quad m=* y_{b} y_{c} y_{n} y_{n-2} y_{a} *+h_{2} .
$$

Proof. It follows from Proposition 10 that the right-hand side asterisk in $m$ stands for a monomial of even length (otherwise $m \in H$ ). According to Lemma 8 we have $\left[y_{1} y_{2}, y_{3} y_{4}, y_{5}\right]=0$. This yields

$$
y_{1} y_{2} y_{3} y_{4} y_{5}-y_{3} y_{4} y_{1} y_{2} y_{5}-y_{5} y_{1} y_{2} y_{3} y_{4}+y_{5} y_{3} y_{4} y_{1} y_{2}=0
$$

Now put $a$ and $b$ instead of the indices 1 and $2, n$ and $n-2$ instead of 3 and 4 , and $c$ instead of 5 , and multiply by $*$ on the left and on the right. Pay attention to the fact that $* y_{c} y_{a} y_{b} y_{n} y_{n-2} *$ and $* y_{c} y_{n} y_{n-2} y_{a} y_{b} *$ lie in $H$ by Proposition 10, since the right-hand $*$ is of even length. This proves the existence of $h_{1}$.

For $h_{2}$ we use the equality $\left[y_{1} y_{2}, y_{3}, y_{4} y_{5}\right]=0$ obtained in Lemma 8 , We substitute the indices 1 and 2 by $n$ and $n-2$, and $3,4,5$ for $a, b, c$, respectively. Then observe that $* y_{n} y_{n-2} y_{a} y_{b} y_{c} * \equiv * y_{a} y_{b} y_{n} y_{n-2} y_{c} *(\bmod H)$ according to the first equality since the right-hand $*$ stands for a monomial of even length. Thus the second and the fourth summand in

$\left[y_{n} y_{n-2}, y_{a}, y_{b} y_{c}\right]=y_{n} y_{n-2} y_{a} y_{b} y_{c}-y_{a} y_{n} y_{n-2} y_{b} y_{c}-y_{b} y_{c} y_{n} y_{n-2} y_{a}+y_{b} y_{c} y_{a} y_{n} y_{n-2}$ are in $H$, and the first and the third give us exactly the second equality.

Lemma 12. Let $m=* y_{a} y_{n} y_{n-2} y_{b} y_{c} * \in\left(P_{n}^{A} / V(n)\right) \backslash H$ be a monomial. Then $m=* y_{c} y_{n} y_{n-2} y_{a} y_{b} *+h_{3}$ for some $h_{3} \in H$. In other words, modulo $H$ one can make cyclic permutations of the letters $y_{a}, y_{b}, y_{c}$ in $m$.

Proof. The right-hand $*$ of $m$ is a monomial of odd length since otherwise $m \in H$. Then we repeat the argument of the second equality in Lemma 11 substituting in $\left[y_{1} y_{2}, y_{3}, y_{4} y_{5}\right]=0$ the indices $1,2,3$ by $a, b, c$, and 4 and 5 by $n$ and $n-2$, respectively. Thus

$$
*\left(y_{a} y_{b} y_{c} y_{n} y_{n-2}-y_{c} y_{a} y_{b} y_{n} y_{n-2}-y_{n} y_{n-2} y_{a} y_{b} y_{c}+y_{n} y_{n-2} y_{c} y_{a} y_{b}\right) *=0 .
$$


The last two summands lie in $H$ since the right-hand side $*$ is of odd length. We transform the first two as follows. The right-hand asterisk above is $*=y_{d} m_{1}$ for some monomial $m_{1}$. So we apply the first equality of Lemma 11 first to $y_{b} y_{c} y_{n} y_{n-2} y_{d}$ and then to $y_{a} y_{b} y_{n} y_{n-2} y_{d}$ :

$$
\begin{aligned}
& * y_{a} y_{b} y_{c} y_{n} y_{n-2} y_{d} m_{1}-* y_{a} y_{n} y_{n-2} y_{b} y_{c} y_{d} m_{1} \in H, \\
& * y_{c} y_{a} y_{b} y_{n} y_{n-2} y_{d} m_{1}-* y_{c} y_{n} y_{n-2} y_{a} y_{b} y_{d} m_{1} \in H,
\end{aligned}
$$

and thus we are done.

Remark 13. We note that in all the above statements we used even permutations of the variables. That is, the resulting monomials will always be even as long as the initial ones are even.

Lemma 14. One has $\operatorname{dim} P_{4}^{A} / V(4)=2^{4-1}-1=7$.

Proof. This was shown to be true in 2]; see the remarks after Conjecture 1.2 in [2].

Lemma 15. Let $m=y_{\sigma(1)} \ldots y_{\sigma(n-3)} y_{n} y_{n-2} y_{\sigma(n)} \in H(n-2, n-1)$ be a monomial. Then

$$
m=y_{1} y_{2} \ldots y_{n-4} y_{n-3} y_{n} y_{n-2} y_{n-1}+h
$$

for some polynomial $h \in H$.

Proof. We induct on $n$. When $n=4$ either $m=y_{1} y_{4} y_{2} y_{3}$ or $m=y_{3} y_{4} y_{2} y_{1}$ and the second cannot happen as it corresponds to an odd permutation. If $n=5$ we have $m=y_{\sigma(1)} y_{\sigma(2)} y_{5} y_{3} y_{\sigma(5)}$. Applying Lemma 11 we permute cyclically $y_{\sigma(1)}, y_{\sigma(2)}$, $y_{\sigma(3)}$ in order to get $\sigma(1)=1$; since $\sigma \in A_{5}$, then $\sigma(2)=2$ and $\sigma(5)=4$.

Letting $n>5$ and $\sigma(j)=1$, we shall show that modulo $H$ one can rewrite $m$ so that $j=1$. Assume $j \geq 2$. According to Lemma 11 we move the block $y_{n} y_{n-2}$ to the left and either $m=* y_{n} y_{n-2} y_{1} *+h_{1}$ or $m=* y_{1} y_{n} y_{n-2} *+h_{1}$. Now by the second part of Lemma 11 we can move $y_{1}$ one or more positions to the left, and so on. If we obtain $m \equiv y_{a} y_{n} y_{n-2} y_{1} y_{b} *(\bmod H)$ we cannot apply Lemma 11, but we use Lemma 12 instead and move $y_{1}$ to the left.

When $\sigma(1)=1$ we apply the induction to the monomial $m_{1}$ where $m=y_{1} m_{1}$. Since $y_{1}$ comes first the monomial $m_{1}$ corresponds to an even permutation of $(2, \ldots, n)$.

Proof. (of Theorem 22). Suppose that $\operatorname{dim} P_{n-1}^{A} / V(n-1)=2^{n-2}-1$ for some $n \geq 5$. By Lemma 15. the vector space $H(n-2, n-1)$ is one dimensional modulo $H$ and is spanned by the monomial $y_{1} y_{2} \ldots y_{n-4} y_{n-3} y_{n} y_{n-2} y_{n-1}$. But we have established that $P_{n}^{A} / V(n)=H(n-2, n-1)+H$; see Corollary 7 . Thus $\operatorname{dim} P_{n}^{A} / V(n) \leq$ $1+\operatorname{dim} H$.

But by the induction we have $\operatorname{dim} H \leq\left(2^{n-2}-1\right)+\left(2^{n-2}-1\right)$; hence

$$
\operatorname{dim} P_{n}^{A} / V(n) \leq 1+\operatorname{dim} H \leq 1+2^{n-1}-2=2^{n-1}-1 .
$$

As we already observed this proves our theorem. In other words one has $V(n)=$ $T(E) \cap P_{n}^{A}$, and thus Henke and Regev's conjecture is true.

\section{ACKNOWLEDGMENTS}

The authors would like to thank the referee, whose valuable comments and advice improved much of the paper. 


\section{REFERENCES}

1. A. Henke, A. Regev, Explicit decompositions of the group algebras $F S_{n}$ and $F A_{n}$, in "Polynomial identities and combinatorial methods" (Pantelleria, 2001), Lecture Notes in Pure and Appl. Math. 235, Dekker, New York, 2003, 329-357. MR2021806 (2004j:20018)

2. A. Henke, A. Regev, A-codimensions and A-cocharacters, Israel J. Math. 133 (2003), 339-355. MR.1968434 (2004b:16029)

3. D. Krakowski, A. Regev, The polynomial identities of the Grassmann algebra, Trans. Amer. Math. Soc. 181 (1973), 429-438. MR0325658 (48:4005)

IMECC, UNICAMP, Cx. P. 6065, 13083-970 CAMPINAS, SP, BraziL

E-mail address: dimasjg@ime.unicamp.br

IMECC, UNICAMP, Cx. P. 6065, 13083-970 CAMPINAS, SP, BRAZIL

E-mail address: plamen@ime.unicamp.br 